\title{
Soft, tough, and flexible curdlan hydrogels and organogels fabricated by covalent cross-linking
}

Yukiko Enomoto-Rogers ${ }^{1,2}$, Satoshi Kimura ${ }^{1}$, and Tadahisa Iwata ${ }^{1, *}$

1. Science of Polymeric Materials, Department of Biomaterial Sciences, Graduate School of Agricultural and Life Sciences, The University of Tokyo, 1-1-1 Yayoi, Bunkyo-ku, Tokyo 113-8657, Japan

\section{National Institute of Advanced Industrial Science and Technology (AIST)}

Eco-renewable materials group, Structural Materials Research Institute

2266-98 Anagahora, Simoshidami, Moriyama-ku, Nagoya, 463-8560, Japan

*Corresponding author. Tel: +81-3-5841-5266; Fax: +81-3-5841-1304.

E-mail: atiwata@mail.ecc.u-tokyo.ac.jp

Keyword: curdlan, hydrogel, organogel, cross-link

\begin{abstract}
Soft, tough and flexible curdlan (CD) hydrogels were fabricated by covalent cross-linking of CD using ethylene glycol diglycidyl ether (EGDGE) as a cross-linker. The mechanical properties of the gels were investigated by uniaxial compression test. CD hydrogels showed non-linear elastic behavior. The elastic modulus of CD hydrogels was 0.004-0.011 kPa. CD hydrogels were highly tough and did not fracture under compression to $90 \%$ strain at the maximum. The gels could retain water during compression, and no water was squeezed out from the gel. In addition, CD hydrogel could recover the original shape and elastic modulus after $90 \%$ compression, indicating its high flexibility. Subsequently, hydroxyl groups of CD hydrogels were acetylated in acetic anhydride and pyridine system to give hydrophobic property, and novel CD organogels were obtained. CD organogel were swollen in chloroform and resistant to repeated drying and swelling treatment in chloroform.
\end{abstract}

\section{Introduction}

Polysaccharides are attractive material for soft material science due to their biocompatibility, biodegradability, or bio-activities [1]. Many kinds of polysaccharides, such as cellulose [2], agarose [3], gellan gum [4], alginate [5], curdlan [6], are known to form hydrogels. Hydrogels are self-standing soft material that is highly swollen in a liquid water and have been extensively studied for many application areas such as medical, food, or bio-enginieering sciences [7-9]. Curdlan (CD) is a polysaccharide composed of $(1 \rightarrow$ 
3)- $\beta$-D-glucose units which is produced by Alcaligenes faecalis.[10-12] CD has high molecular weight more than a couple tens $\mathrm{kDa}$, and attractive bio-based material that can be obtained from nature [1]. CD forms a gel by heating and are used in cosmetics or food industry [13-16]. However, most of hydrogels of polysaccharideare nomally very brittle and have low mechanical strength, which has been restricting an application of polysaccharide-based hydrogels as industrial material. Improvement and designing of mechanical properties of hydrogels, such as toughness or elasticity is crucial issue to utilize polysaccharide hydrogels for biomedical or engineering application.

There have been some reports on polysaccharide-based hydrogels with high mechanical properties. Double network gels consisting of gelatin and cellulose are known to have high toughness and high elasticity, and they are expected to be suitable for artificial tendon or cartilage which are categolized as hard tissue [17]. Interpenetrating hydrogels consisting of alginate and polyacrylamide have also been reported as a soft hydrogels with high mechanical properties [18, 19]. Polyacrylamide are widely used as a contact lenses and its further application for biomedical field is expected, but toxicity of acrylamide monomer has been concerned. Regarding the mechanical property of hydrogels, polysaccharide-based gel that has softness, toughness and flexibility at the same time, has not been reported to our knowledge. Such kind of hydrogels would be novel class of bio-based hydrogels and could be applicable for artificial soft tissue in tissue-engineering or regeneration studies.

Chemical crosslinking is an one of the effective way to improve mechanical strength and water retention ability of the gels [19-23]. Ethylene glycol diglycidyl ether (EGDGE) is avairable as a crosslinking reagent for polysaccharides,and is suitable reagent for biomedical application because it is amphiphilic and can be removed from the human body [22, 24]. Herein, we describe preparion of covalently cross-linked CD hydrogels with soft, tough and flexible properties, and demonstrated their excellent elastic behavior to repeated compressing. Furthermore, CD organogels are obtained by acetylation of CD hydrogels and their unique swelling behavior for repeated drying and sweling treatments are also described.

\section{Experimental}

\subsection{Materials}

CD was purchased from Wako Pure Chemical Industries, Ltd (Japan). Ethylene glycol diglycidyl ether (EGDGE) and sodium hydroxide $(\mathrm{NaOH})$ were purchased from Tokyo Chemical Industry Co., Ltd. (Japan), and Kanto Chemical Co., Inc. (Japan), respectively. All reagents were used without further purification.

\subsection{Fabrication of $C D$ hydrogels}

The representative procedure for fabrication of CD hydrogel (CDg-1) is as follows; To a $4 \% \mathrm{NaOH}$ aqueous solution $(25 \mathrm{~mL})$ of $\mathrm{CD}(1.0 \mathrm{~g})$, EGDGE (1.0 ml, 1.1 molar equivalent to 
anhydroglucose unit (AGU)) was added dropwise. A solution was stirred for $1 \mathrm{~min}$ and poured into a glass mold with $10 \mathrm{~mm}$ diameter and $10 \mathrm{~mm}$ height, and set at room temperature to allow gel formation for 1 day. The obtained cross-linked gels were removed from the mold in distilled water $(1 \mathrm{~L})$, and then, acetic acid was added to neutralize $\mathrm{NaOH}$. The gels were further washed for 2 days with distilled water to remove residual reagents. The CDg-2 and -3 were prepared by varying concentration of CD and EGDGE as listed in Table 1. The molar ratio of $\mathrm{NaOH}$ to EGDGE was set to 4 molar equivalent.

\subsection{Fabrication of agar hydrogel}

Agar gels were fabricated as follows: Agar (2 g) was dissolved hot distilled water (98 $\mathrm{ml}$ ) and poured into a mold with $10 \mathrm{~mm}$ diameter and $10 \mathrm{~mm}$ height, and set at room temperature for 3 hours to allow gel formation.

\subsection{Fabrication of organogels}

The hydrogels were immersed in ethanol and the media was subsequently exchanged by acetic anhydride/ pyridine. The gels in acetic anhydride/ pyridine mixed media were heated at $70^{\circ} \mathrm{C}$ for 3 days. The obtained organogels were washed by chloroform to remove acetic anhydride and pyridine.

\subsection{Media composition of the gels}

The media composition of the gels was calculated from the weights of the swollen gel and the dried gel; The media composition $($ wt \%) $=(1-$ [dried gel] $/$ [swollen gel] $) \times 100$. Dried hydrogels and organogels were obtained by freeze-drying or vacuuming.

\subsection{Compression test}

Compressive test was carried using EZ-test (Shimadzu, Japan) to measure mechanical properties of the hydrogels. The hydrogels were compressed perpendicular to the height direction by parallel metal plates connected to a load cell at $1 \mathrm{~mm} / \mathrm{min}$ at room temperature. The elastic modulus $(E)$ was determined by the average slope over the strain ratio range of $10-20 \%$ in the stress-strain curve which was considered as a linear region. To determine elastic modulus, five specimens were used and the data were averaged for each gel. Strain rates (\%) were referenced to the initial height of the gel. For the repeated compressing test, the one specimen was used for five times to obtain strain-stress curves, and the recovery rates (\%) were calculated as the relative height of the CD gel after compressing to its original height.

\section{Results and Discussion}

\subsection{Fabrication of CD hydrogels}


The CD was cross-linked using EGDGE in NaOHaq. solution as described in Figure 1. EGDGE is considered to form ether bond between epoxy group and hydroxyl groups of CD in alkaline solution. Three different CD hydrogels, namely, CDg-1, -2 , and -3 were obtained by varying the amount of EGDGE as listed in Table 1. As can be seen in the images in Figure 2, the self-standing hydrogels were successfully obtained and the gels prepared with higher EGDGE concentration formed more swollen gel in the order of CDg-1 $>-2>-3$. Water composition of CDg-1, -2, and -3 was 97.2, 95.4, and $94.8 \%$ respectively, and decreased in the order of CDg-1 $>-2>-3$ with decrease in EGDGE (Table 1). This result is opposite to the common tendency that media composition of a gel decreases with increase in cross-linker [25, 26]. We attribute this result to poor cross-linking efficiency caused by steric hindrance that occurs when more cross-linker was added. The relative molar amount of EGDGE to AGU of CD for CDg-1, -2 , and -3 is 1.1, 0.4 and 0.2 , respectively. In the case of CDg-1, all AGU is theoretically substituted by at least one epoxy group. There might be steric hindrance for next cross-linking reaction between another epoxy group and hydroxyl group of different CD chain, and as a result, free hydroxyl group was formed by cleavage of epoxy group. This might be one reason for the result that media composition became higher when more cross-linker was loaded.

\subsection{Compressive mechanical properties of CD hydrogels}

Compressive mechanical property of CD hydrogels was investigated by uniaxial compression test. Figure 3 shows stress-strain curves of CD hydrogels under uniaxial compression to $50 \%$ and $90 \%$ strain. CD hydrogels showed non-linear elastic behavior. The gels were quite tough and fracture stress could not be determined. The elastic modulus of CD hydrogels is listed in Table 2 with a comparison with other polysaccharide-based gels or gelatin gels. The elastic modulus of CD-1, -2 , and -3 hydrogels were $0.004,0.006$, and $0.011 \mathrm{MPa}$, respectively. Elastic modulus tended to increase in the order of CDg- $1<-2<-3$ with a decrease in EGDGE. This tendency agreed well with the result that water composition of the gels decreased with a decrease in EGDGE, likely because the cross-linking efficiency was low when more EGDGE was added, as discussed above. The elastic modulus values are as low as those of other polysaccharide or gelatin gels, indicating that CD hydrogels were as soft as common polysaccharide-based hydrogels.

Interestingly, CDg-1 was able to sustain a compressive strain of $90 \%$ as well as $50 \%$ with full strain recovery (Figure 4a). In the case of CDg-2 and CDg-3, these gels were also able to recover original shape from 50\% compression (Figures $4 \mathrm{~b}$ and $4 \mathrm{c}$ ). CDg-2 and CDg-3 gels did not fracture after 90\% compression, although the gels were irreversibly deformed, suffering from hysteresis. It is noteworthy that all CD hydrogels retained water during compression and no water was squeezed out from the gel at $90 \%$ strain. Agar hydrogel was compressed for comparison, but it broke easily at 50\% compression, as shown in Figure $4 \mathrm{~d}$. 
It was found that CD hydrogels exhibited high toughness in addition to softness.

\subsection{Repeated compression test}

The flexibility of CD hydrogels was investigated by repeating compression for five times. The recovery rates of CD hydrogels after compressing to 25, 50 and $90 \%$ were plotted as shown in Figure 5. CD hydrogels showed high recovery rate after 25\% and 50\% compression. The recovery rates were $100 \%$ for all gels after $25 \%$ compression for five times and the elastic modulus of the gels showed no substantial decrease after five times compression. CDg-1 and -2 showed $100 \%$ recovery after one compression to $50 \%$ strain, and then showed small decrease in recovery rate after 4 or 3 times compression. In the case of CDg-3, the recovery rate after $50 \%$ compression was $97 \%$, which is indicative to hysteresis. Elastic modulus of CDg-3 slightly dropped after one compression, as suggested in its strain-stress curves in Figure 3c. It should be stressed that CDg-1 recovered 90-96\% of the original height after repeated compressing to $90 \%$ strain (Figure 5a). CDg-1 showed no change in the elastic modulus after repeated $90 \%$ compressing for five times, as suggested in Figure 3d. CDg-2 and -3 suffered from hysteresis and irreversibly deformed after 90\% compression for one time as discussed in the previous section.

It was revealed that CD hydrogels were soft, tough and flexible gel. CD hydrogels exhibited low elastic modulus of 0.004-0.011 MPa and could sustain 90\% strain with 90-100\% recovery. To our knowledge, there has been no report on the polysaccharide-based hydrogels that has these three properties at the same time. Generally, polysaccharide-based gels, such as agar, are soft but not tough or flexible. Polysaccharide-based gels have poor mechanical property and fracture at ca. 20-60\% strain with low compression force [17]. Bacteria cellulose hydrogel has been reported to exhibit non-linear elastic behavior similar to those of CD hydrogels, but it could not sustain water during compression and suffers from hysteresis after compression [17]. Regarding polysaccharide-based tough and flexible gel, there has been a report on double network gel consisting of gelatin and cellulose [17]. Their elastic modulus was 1-3 MPa and their maximum compressive strain was ca. $40 \%$. CD hydrogels were superior to common polysaccharide-based hydrogels in its toughness and flexibility and different from double network gels in its softness. CD hydrogels would be novel class of polysaccharide-based hydrogel that has softness, toughness, and flexibility at the same time.

\subsection{Fabrication of CD organogels}

CD has no solubility in most organic solvents such as chloroform. It is reported that hydroxyl groups of CD can be substituted by ester groups and that the properties of CD esters turned to be hydrophobic and organic solvent-soluble [27]. In order to give novel properties to CD-based gels, we have fabricated CD organogels by acetylation of remaining hydroxyl 
groups of CD gels as described in Figure 1. After exchanging the media of the hydrogels from water to pyridine, CD gels were treated by acetic anhydride and pyridine. The CD gels kept swollen during acetylation in the media, and remained swollen in pyridine under dry condition at room temperature. The media of the CD orgnogels could be easily exchanged from pyridine to chloroform. The volume of CD organogel in chloroform was higher compared to the hydrogels and chloroform composition reached ca. $99 \%$ for all CD gels as listed in Table 1. In the IR spectrum of CD organogels, the absorption band of $\mathrm{C}=\mathrm{O}$ bond derived from acetyl group was observed at $1750 \mathrm{~cm}^{-1}$ and no absorption of hydroxyl groups were observed at ca.3500 $\mathrm{cm}^{-1}$, indicating successful acetylation (Data not shown). It has been reported that polysaccharide hydrogels could not recover to original swollen state completely once they were dried [23]. The CD organogel were resistant to repeated drying and swelling treatment in chloroform. As shown in Figure 6, dried CD organogel could be re-swollen in chloroform, and its media composition recovered original value. CD organogels would be novel material that is stable under dry condition or high temperature.

\section{Conclusion}

CD hydrogels were fabricated by covalent cross-linking using EGDGE as a cross-linker. The compressive mechanical tests revealed that CD hydrogels were soft, tough and flexible gel. CD hydrogels showed non-linear elastic behavior, and their elastic modulus was 0.004-0.011 MPa. CD hydrogels sustained compressive strain of $90 \%$ at the maximum and showed recovery rate of more than ca.95-100\% after compression. CD hydrogels retained water during compression, and no water was squeezed out from the gel. CD hydrogels were subsequently acetylated in acetic anhydride/ pyridine system and the novel CD organogel were successfully obtained. CD organogels were swollen in chloroform and resistant to repeated drying and swelling treatment. CD hydrogels would be applicable for artificial soft tissue in tissue-engineering or regeneration studies. CD organogels could be applied to wide range of soft material science, due to its organic solvent absorbency, heat resistance or dryness resistance properties in future.

\section{Acknowledgements}

This study was supported by JST-ALCA, Grant-in-Aid for Scientific Research (A) (No. 26248044) (to T.I.) and Grant-in-Aid for Young Scientists (B) (No.26850108) (to Y.E-R) from the Japan Society for the Promotion of Science (JSPS).

\section{References}

[1] Garcia-Gonzalez CA, Alnaief M, Smirnova I. Polysaccharide-based aerogels-Promising biodegradable carriers for drug delivery systems. Carbohydrate Polymers. 2011;86:1425-38. 
[2] Fei B, Wach RA, Mitomo H, Yoshii F, Kume T. Hydrogel of biodegradable cellulose derivatives. I. Radiation-induced crosslinking of CMC. Journal of Applied Polymer Science. 2000;78:278-83.

[3] Normand V, Lootens DL, Amici E, Plucknett KP, Aymard P. New insight into agarose gel mechanical properties. Biomacromolecules. 2000;1:730-8.

[4] Morris ER, Nishinari K, Rinaudo M. Gelation of gellan - A review. Food Hydrocolloids. 2012;28:373-411.

[5] Augst AD, Kong HJ, Mooney DJ. Alginate hydrogels as biomaterials. Macromol Biosci. 2006;6:623-33.

[6] Nishinari K, Takahashi R. Interaction in polysaccharide solutions and gels. Current Opinion in Colloid \& Interface Science. 2003;8:396-400.

[7] Lee KY, Mooney DJ. Hydrogels for tissue engineering. Chemical Reviews. 2001;101:1869-79.

[8] Shoichet MS. Polymer Scaffolds for Biomaterials Applications. Macromolecules. 2010;43:581-91.

[9] Chang C, Zhang L. Cellulose-based hydrogels: Present status and application prospects. Carbohydrate Polymers. 2011;84:40-53.

[10] Harada T, Misaki A, Saito H. Curdlan: A bacterial gel-forming 8-1, 3-glucan. Archives of Biochemistry and Biophysics. 1968;124:292-8.

[11] Nobutami K, Tokuya H. Ultrastructure of Curdlan. Fiber Diffraction Methods: AMERICAN CHEMICAL SOCIETY; 1980. p. 363-83.

[12] Tokuya H. Production, Properties, and Application of Curdlan. Extracellular Microbial Polysaccharides: AMERICAN CHEMICAL SOCIETY; 1977. p. 265-83.

[13] Nishinari K, Zhang H. Recent advances in the understanding of heat set gelling polysaccharides. Trends in Food Science \& Technology. 2004;15:305-12.

[14] Nobe M, Dobashi T, Yamamoto T. Dynamics in dialysis process for liquid crystalline gel formation. Langmuir. 2005;21:8155-60.

[15] Tada T, Matsumoto T, Masuda T. Dynamic viscoelasticity and small-angle X-ray scattering studies on the gelation mechanism and network structure of curdlan gels. Carbohydrate Polymers. 1999;39:53-9.

[16] Zhang HB, Nishinari K, Williams MAK, Foster TJ, Norton IT. A molecular description of the gelation mechanism of curdlan. International Journal of Biological Macromolecules. 2002;30:7-16. [17] Nakayama A, Kakugo A, Gong JP, Osada Y, Takai M, Erata T, et al. High mechanical strength double-network hydrogel with bacterial cellulose. Advanced Functional Materials. 2004;14:1124-8. [18] Omidian H, Rocca JG, Park K. Elastic, superporous hydrogel hybrids of polyacrylamide and sodium alginate. Macromolecular Bioscience. 2006;6:703-10.

[19] Sun J-Y, Zhao X, Illeperuma WRK, Chaudhuri O, Oh KH, Mooney DJ, et al. Highly stretchable and tough hydrogels. Nature. 2012;489:133-6.

[20] Abdurrahmanoglu S, Can V, Okay O. Design of high-toughness polyacrylamide hydrogels by hydrophobic modification. Polymer. 2009;50:5449-55. 
[21] Darnell MC, Sun J-Y, Mehta M, Johnson C, Arany PR, Suo Z, et al. Performance and biocompatibility of extremely tough alginate/polyacrylamide hydrogels. Biomaterials. 2013;34:8042-8.

[22] Liang S, Wu J, Tian H, Zhang L, Xu J. High-Strength Cellulose/Poly(ethylene glycol) Gels. Chemsuschem. 2008;1:558-63.

[23] Chang C, Duan B, Zhang L. Fabrication and characterization of novel macroporous cellulose-alginate hydrogels. Polymer. 2009;50:5467-73.

[24] Kono H, Zakimi M. Preparation, Water Absorbency, and Enzyme Degradability of Novel Chitin- and Cellulose/Chitin-Based Superabsorbent Hydrogels. Journal of Applied Polymer Science. 2013;128:572-81.

[25] Dash R, Foston M, Ragauskas AJ. Improving the mechanical and thermal properties of gelatin hydrogels cross-linked by cellulose nanowhiskers. Carbohydrate Polymers. 2013;91:638-45. [26] Sannino A, Madaghiele M, Conversano F, Mele G, Maffezzoli A, Netti PA, et al. Cellulose derivative-hyaluronic acid-based microporous hydrogels cross-linked through divinyl sulfone (DVS) to modulate equilibrium sorption capacity and network stability. Biomacromolecules. 2004;5:92-6.

[27] Marubayashi H, Yukinaka K, Enomoto-Rogers Y, Takemura A, Iwata T. Curdlan ester derivatives: Synthesis, structure, and properties. Carbohydrate Polymers. 2014;103:427-33.

\section{Figure Legend}

Figure 1. Fabrication scheme and representative chemical structure of covalently cross-linked curdlan (CD) hydrogel and organogel.

Figure 2. Images of cross-linked CD hydrogels.

Figure 3. Compressive strain-stress curves of (a) (d) CDg-1, (b) (e) CDg-2, and (c) (f) CDg-3 with repeated compression for five times. (a) (b) (c) $50 \%$ and (d) (e) (f) $90 \%$ strain. Numbers indicate measuring time.

Figure 4. Images of (a) CDg-1, (b) CDg-2, (c) CDg-3, and (d) agar hydrogels during and after compression to $0 \%, 50 \%$ and $90 \%$.

Figure 5. Recovery rate of (a) CDg-1, (b) CDg-2 and (c) CDg-3 after repeated compression to 25\% ( $\triangle$ ), 50\% ( $\square$ ) and $90 \%(\bigcirc)$ strain.

Figure 6. Representative images of CDg-3 organogel.

Table 1. Characteristics of cross-linked CD gels.

Table 2. Compressive properties of hydrogels. 


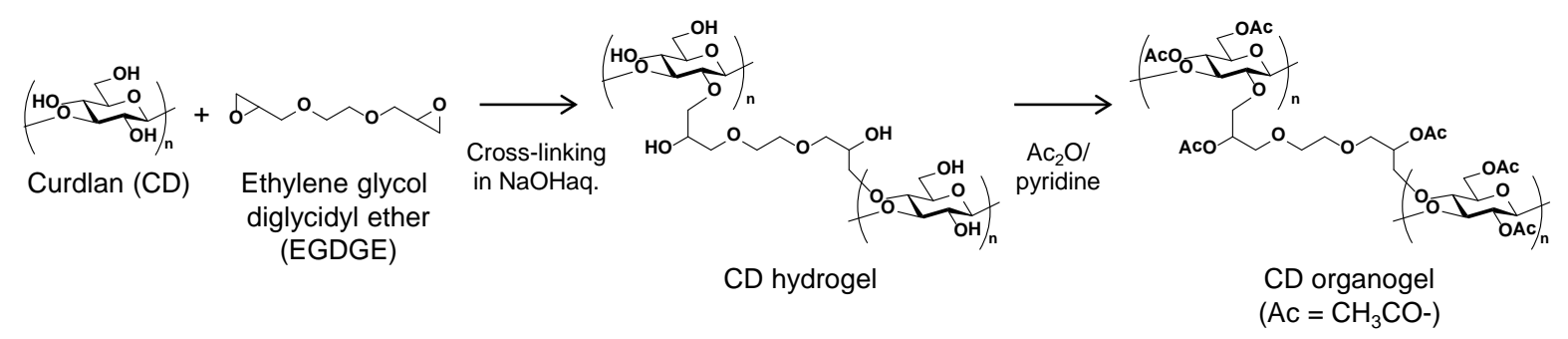

Figure 1. Fabrication scheme and representative chemical structure of covalently cross-linked curdlan (CD) hydrogel and organogel. 


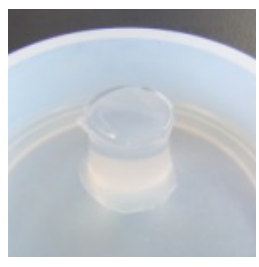

CDg-1

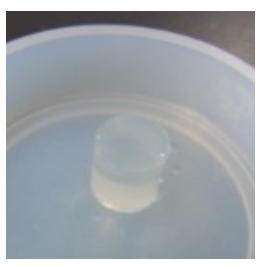

$\mathrm{CDg}-2$

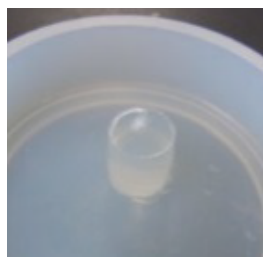

CDg-3

Figure 2. Images of cross-linked CD hydrogels. 

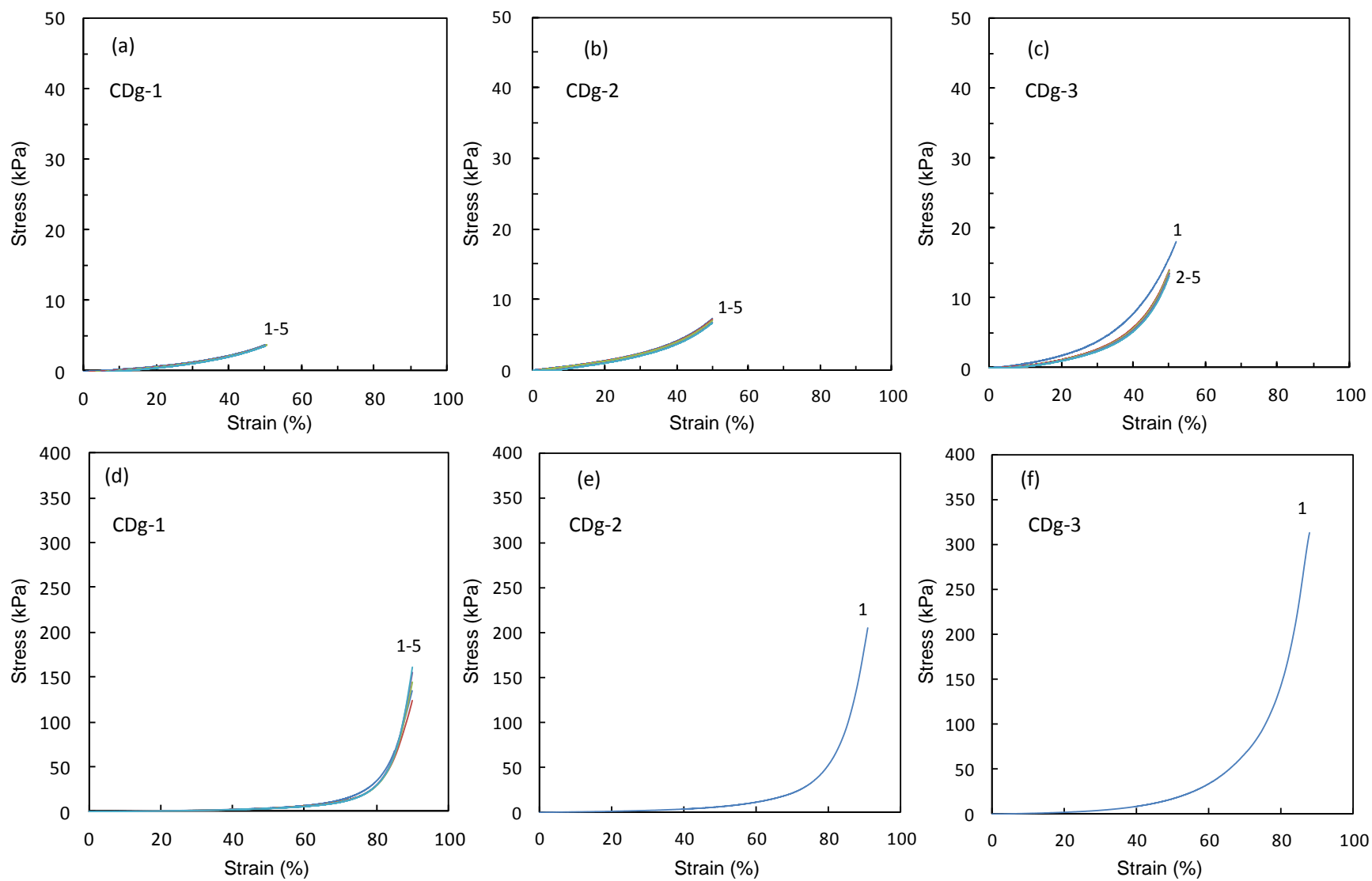

Figure 3. Compressive strain-stress curves of (a) (d) CDg-1, (b) (e) CDg-2, and (c) (f) CDg-3 with repeated compression for five times. (a) (b) (c) $50 \%$ and (d) (e) (f) $90 \%$ strain. Numbers indicate measuring time. 
(a)
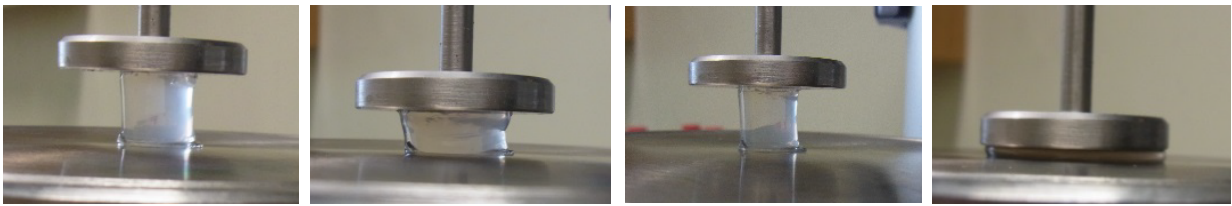

(b)
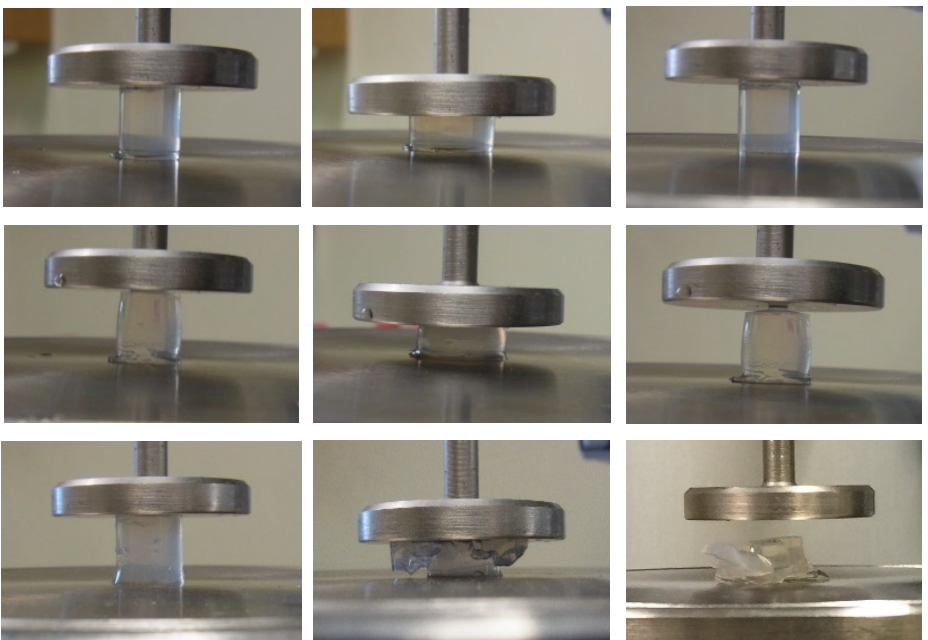

$50 \%$ compression
After $50 \%$ compression

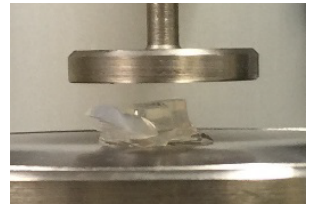

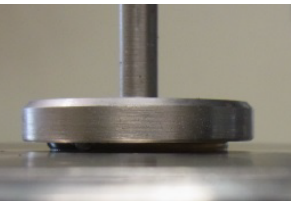
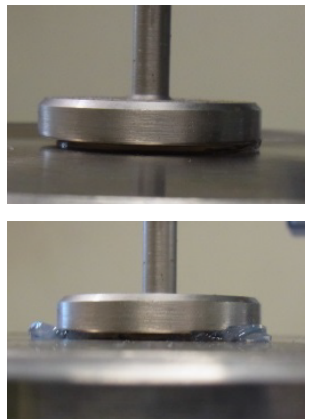

$90 \%$ compression
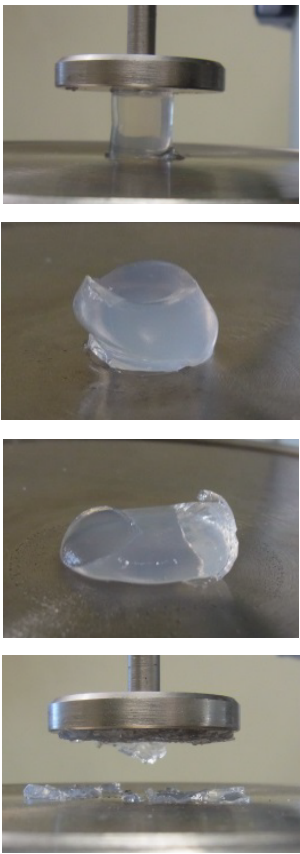

After $90 \%$ compression

Figure 4. Images of (a) CDg-1, (b) CDg-2, (c) CDg-3, and (d) agar hydrogels during and after compression to $0 \%, 50 \%$ and $90 \%$. 

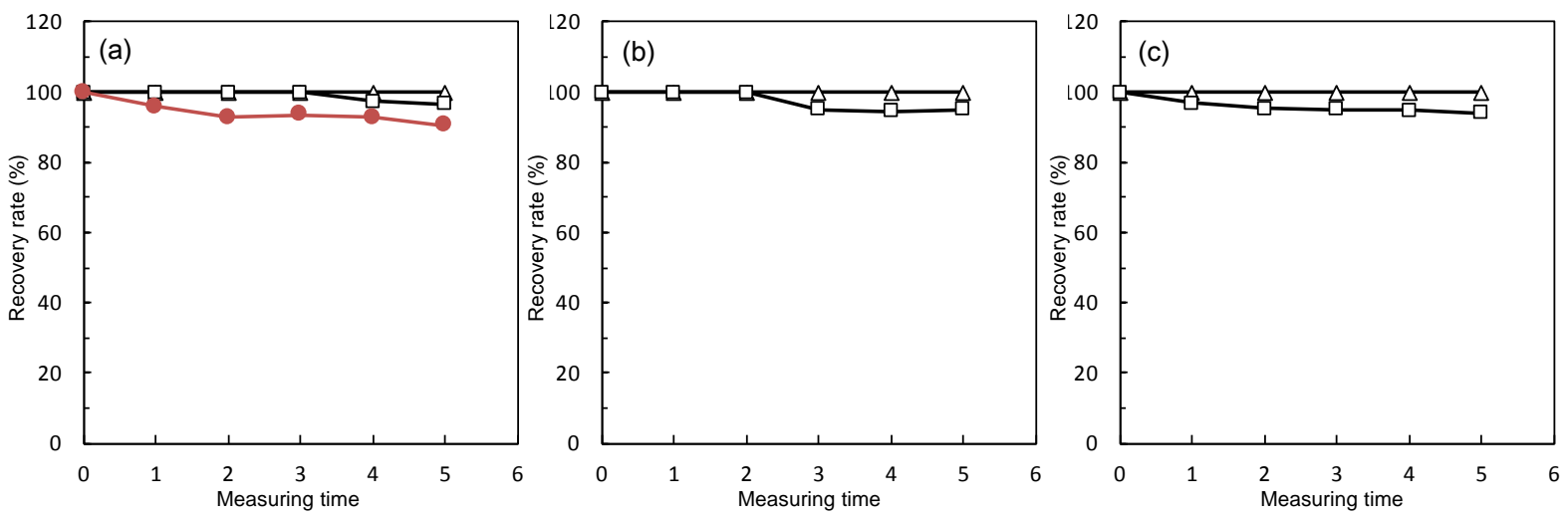

Figure 5. Recovery rate of (a) CDg-1, (b) CDg-2 and (c) CDg-3 after repeated compression to 25\% ( $\triangle$ ), $50 \%(\square)$ and $90 \%(\bigcirc)$ strain. 
Table 1. Characteristics of cross-linked CD gels

\begin{tabular}{ccccccccc}
\hline Gels & $\mathrm{CD}(\mathrm{g})$ & $\begin{array}{c}\mathrm{H}_{2} \mathrm{O} \\
(\mathrm{mL})\end{array}$ & $\begin{array}{c}\mathrm{EGDGE} \\
(\mathrm{mL})\end{array}$ & $\begin{array}{c}\mathrm{EGDGE}] \\
{[\mathrm{AGU}]} \\
(\mathrm{mol} / \mathrm{mol})\end{array}$ & $\begin{array}{c}\mathrm{NaOH} \\
(\mathrm{g})\end{array}$ & $\begin{array}{c}\text { Water } \\
\text { composition } \\
\text { of hydrogels } \\
(\text { wt \%) }\end{array}$ & $\begin{array}{c}\mathrm{CHCl}_{3} \\
\text { composition } \\
\text { of } \\
\text { organogels } \\
\text { (wt \%) }\end{array}$ & $\begin{array}{c}\mathrm{CHCl}_{3} \\
\text { composition } \\
\text { of re-swollen } \\
\text { organols } \\
\text { (wt \%) }\end{array}$ \\
\hline $\mathrm{CDg}-1$ & 1.0 & 25 & 1.0 & 1.1 & 1.0 & 97.2 & 99.5 & 99.2 \\
$\mathrm{CDg}-2$ & 1.0 & 25 & 0.4 & 0.4 & 0.4 & 95.4 & 99.0 & 99.2 \\
$\mathrm{CDg}-3$ & 1.0 & 25 & 0.2 & 0.2 & 0.2 & 94.8 & 98.1 & 98.2 \\
\hline
\end{tabular}


Table 2. Compressive properties of hydrogels

\begin{tabular}{cccc}
\hline Samples & $\begin{array}{c}\text { Elastic } \\
\text { modulus } \\
(\mathrm{MPa})\end{array}$ & $\begin{array}{c}\text { Fracture } \\
\text { stress } \\
(\mathrm{MPa})\end{array}$ & $\begin{array}{c}\text { Fracture } \\
\text { strain (\%) }\end{array}$ \\
\hline CDg-1 & 0.004 & - & - \\
$\mathrm{CDg}-2$ & 0.006 & - & - \\
CDg-3 & 0.011 & - & - \\
Bacteria cellulose $^{17}$ & 0.007 & - & - \\
Agarose & 0.28 & 0.013 & 20 \\
Gellan gum ${ }^{17}$ & 0.62 & 0.47 & 60 \\
${\text { Gelatin } 15 \mathrm{wt} \%{ }^{17}}^{17}$ & 0.047 & 0.029 & 34 \\
\hline
\end{tabular}




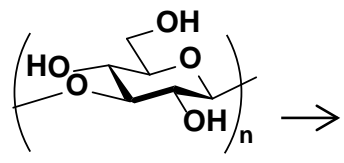

Curdlan (CD) Crosslinking<smiles>C(COCC1CO1)OCC1CO1</smiles>

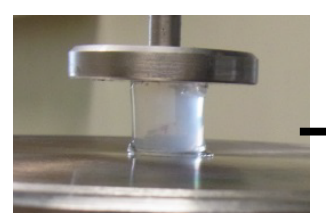

CD hydrogel

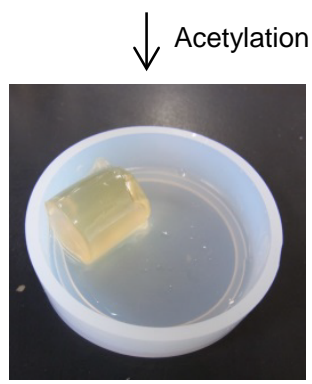

CD acetate organogel in chloroform

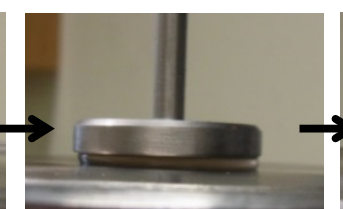

$90 \%$ compression

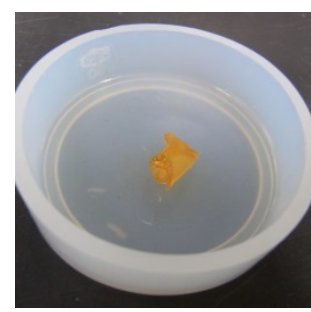

Dried

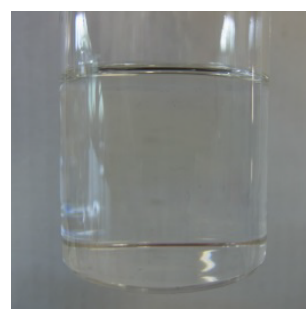

re-swollen in chloroform 